\title{
Monitoring and Planning Capacities in the Acquisition of Music Performance Skills
}

\author{
CAROLINE PALMER, Obio State University \\ CAROLYN DRAKE, CNRS URA316 and University of Paris $V$
}

\begin{abstract}
We investigate changes in cognitive capacities that occur as musicians acquire performance skills. Previous studies suggest that skilled behaviour is characterized by advanced abilities to monitor one's own performance or to plan upcoming events. We first report a study of child pianists (aged 6-14 years old) of beginning and intermediate skill levels who performed well-learned musical pieces. Computer-detected pitch errors in the performances provided evidence for skill-related increases in both monitoring and planning: Children with more musical training showed quicker detection and correction of errors, more anticipatory and less perseveratory behavior, and larger range of planning than children with less training. Next we report a study of adult musicians' advanced performances, which showed heightened sensitivity to melody and repeated pitch structures. Planning and monitoring capacities increased most during initial stages of musical skill acquisition, whereas sensitivity to musical structure increased across all skill levels.
\end{abstract}

Résumé Nous avons étudié les changements dans les habiletés cognitives qui se produisent lorsque les musiciens acquièrent des compétences d'exécution. Des études antérieures suggèrent que le comportement spécialisé se caractérise par des aptitudes avancées à contrôler sa propre exécution ou à planifier les événements à venir. Nous avons d'abord effectué une étude auprès d'enfants pianistes (âgés de 6 à 14 ans), de niveaux débutant et intermédiaire, qui exécutaient des pièces musicales bien apprises. La détection par ordinateur d'erreurs de tons dans l'exécution nous a fourni la preuve de l'augmentation des compétences, tant dans le contrôle que dans la planification; les enfants possédant davantage de formation musicale détectaient et corrigeaient plus rapidement leurs erreurs, démontraient un comportement plus préventif et moins persévérant, ainsi qu'une plus grande capacité de planification que les enfants possédant moins de formation. Nous avons ensuite effectué une étude d'exécution avancée chez des musiciens adultes. Elle a révélé une sensibilité élevée à la mélodie et des schémas tonaux répétés. Les capacités de contrôle et de planification ont augmenté davantage au cours des premières phases d'acquisition de compétences musicales, tandis que la sensibilité à la structure musicale a augmenté à tous les niveaux de compétence.

Although interview studies have addressed social and motivational factors underlying the acquisition of musical skill (Sloboda \& Howe, 1991; Sosniak, 1985), there are surprisingly few empirical studies of cognitive factors (Bamberger, 1986). This article examines the cognitive changes underlying the acquisition of music performance skills. We examine child pianists' achieved skill levels during the first two to eight years of musical training, and we contrast the cognitive factors underlying their performances with those of advanced adult music performances. Despite the well-documented nature of skilled adult music performance (for a review, see Palmer, 1997), there has been relatively little comparison of child and adult performance skills. We focus here on three types of cognitive factors underlying skill acquisition in child and adult performance: monitoring, planning, and awareness of musical structures.

One hallmark of skilled performance in music, speech, and other complex actions is the ability to monitor, or to detect deviations from the intended behaviour and adjust for them. An example is a musician's recovery from a memory lapse during a public recital. Theories of monitoring have been proposed for language production in which speakers compare their own productions to their original intentions and detect any structural deviations (Boomer, 1965; Levelt, 1983). The function of the monitor is to create instructions for repair when deviations between productions and intentions are found. When trouble is detected, corrective action is taken, based on information to which the producer has access. Internal monitoring mechanisms have been proposed that detect and delete erroneous speech patterns such as perseverations in a planned utterance (Shattuck-Hufnagel, 1979). The ability to monitor one's own performance also 
underlies musical skill, according to some music educators and researchers (Seashore, 1938). Pedagogical techniques such as Suzuki music training methods focus on monitoring skills by weighting aural (auditory feedback) methods of teaching more heavily than traditional notation-based methods (Suzuki, 1983). Study of speech errors suggests that monitoring shows increased sensitivity at constituent boundaries, or positions of structural importance (Levelt, 1983). Thus, musicians of advanced skill levels should demonstrate better monitoring skills - quicker detection and correction of errors - than those of less advanced skill levels, especially at musical constituent boundaries.

Another hallmark of skilled performance is the ability to plan, or to construct in advance mental representations of the behaviour to be produced. Plans can account for both fluency, the ability to perform in a smooth and consistent manner, and flexibility, the ability to generate different actions that are functionally equivalent at some level (MacKay, 1982). The ability to form plans increases with practice, and plans can account for both anticipatory and perseveratory behavior. For example, musicians tend to produce pitches too early or too late in a melody (Palmer \& van de Sande, 1995), and speakers often produce a word too soon or too late in an utterance (cf. Garrett, 1980). In fact, studies of errors made during speech and music performance suggest that the range of elements accessible during planning is a function of both the structural relations and serial proximity between elements (Garcia-Albea, del Viso, \& Igoa, 1989; Gruson, 1988; Palmer \& van de Sande, 1995). Experience and practice often lead to more future-oriented (anticipatory) than past-oriented (perseveratory) speech errors (Dell, Berger, \& Svec, 1997; Stemberger, 1989). Thus, skilled musicians' performance may be characterized by plans that incorporate more future-oriented events as well as events from larger ranges surrounding the event currently being produced, than less skilled performance.

Skilled performance in many domains also exhibits heightened sensitivity to structural relationships among sequence elements. Many types of musical structure, such as meter and melody, are emphasized by skilled performers. For example, performers often emphasize strong metrical beats by lengthened durations and delayed onsets (Henderson, 1936), and the melody (the primary voice among notated simultaneities) is often emphasized by dynamic level or early onsets (asynchronies) (Palmer, 1989, 1996; Rasch, 1979). Metrical and melodic structure can influence production errors as well. Pianists playing multi-voiced music from memory made fewer errors in the melody (Palmer \& van de Sande, 1993), and children and adult pianists made more errors in short melodies when metrical, rhythmic grouping, and melodic contour accents were misaligned (Drake, Dowling, \& Palmer, 1991). Thus, musicians of advanced skill levels should demonstrate increased sensitivity to (and greater accuracy for) melodic and metrical structure relative to novices; in particular, errors may occur less often on musical events aligned with the melodic voice or strong metrical accents.

Skilled performers' increased sensitivity to important structural events may be accompanied by decreased sensitivity to less important events. An example is repeating events that are presented successively, such as musical pitches in a melody. Later instances of repeating pitches are often less important on several musical dimensions such as metrical accent, melodic contour, and harmonic content. Speakers' failure to identify and recall repeating linguistic units that are presented visually (called repetition blindness) or auditorily (called repetition deafness) has been demonstrated for repeating letters in words (MacKay, 1969), repeating words in lists and sentences (Kanwisher, 1987; Miller \& MacKay, 1994), and even for semantically identical words across languages (MacKay \& Miller, 1994). Likewise, speech production errors often occur on repeated instances of a phoneme or word, especially deletions of the second of two occurrences of the same element in an utterance (Shattuck-Hufnagel, 1979). We test here for repetition failures in music performances, in which pitch structure typically contains a higher rate of item repetition than is commonly found in speech. Musicians of advanced skill levels may exhibit more errors on item repetitions because of decreased sensitivity for less important events. The second instances of a repeating item especially may be more prone to error in skilled than novice performances because they are perceived as less structurally significant.

We compare monitoring, planning, and structural sensitivity in child and adult music performances in terms of what happens when performance breaks down. Errors, or breakdowns resulting in unintended output, provide a measure of processing that indicates what alternative information or knowledge was accessible during performance. For example, speech errors often reflect a combination of information, such as the resulting word blend "kype" when attempting to say "kind" or "type" (ShattuckHufnagel, 1979), and music production errors often reflect related harmonic information, such as note substitutions in chords (Palmer \& van de Sande, 1993). Blends and other errors are taken as evidence of competing sources of information that are available at a given time (Garrett, 1975; Norman, 1981). We attempt to separate production errors from those due to input demands (failures that may occur during perception or learning) by using welllearned, familiar music that each performer has practiced for several weeks.

Music performance offers several advantages in the study of production errors over other domains. Computer-monitored musical instruments allow examination of performance free of perceptual biases that can arise 
in acoustic domains. Also, the problem of identifying the intentions of a performer can be overcome by the presence of a musical score. Finally, chance estimates for different behavioral outcomes (the likelihood of a given error type occurring by chance) can be computed from statistical properties of Western tonal music that are evidenced in compositional scores. Because the musical complexity tends to be greater for adults' than for childrens' pieces, we analyze the patterns of performance errors relative to chance estimates computed from the statistical characteristics of each musical piece.

One possible reason for the paucity of experimental studies devoted to musical skill acquisition is the difficulty of separating differences due to skill level from those due to task level in non-expert performance. For example, advanced musicians typically play more complex music than beginners, and the complexity differences can overshadow skill level differences. To overcome this problem, we employ a methodology that controls for task difficulty by contrasting an objective measure of difficulty for each musical piece, obtained from experts (music instructors), with observed error rates in the performances of those pieces. Performers are then classified into skill levels on the basis of experts' ratings of difficulty.

We contrast performances of child pianists at beginning and intermediate skill levels - between two and eight years of musical training - to evaluate the development of monitoring and planning capacities. Specifically, we expect performance errors of more advanced pianists to be more quickly detected and corrected, according to monitoring predictions, and more anticipatory behavior and interacting elements that span larger ranges of material, according to planning predictions. Next, we examine advanced adult pianists' performances in terms of monitoring and planning abilities. We also contrast all skill levels for increased sensitivity to melodic and metrical structure and decreased sensitivity to item repetition. In addition, we distinguish effects of musical training, practice, and age on performance at each skill level.

\section{Experiment 1:}

\section{METHOD}

\section{Beginner and Intermediate Skill Levels}

\section{Participants}

29 children between 6 and 14 years of age who had been studying piano for at least one year (ranging from 2 to 8 years) were recruited through local music teachers' associations. The 15 beginners (mean age $=10.2$ years) had been taking private instruction on piano for a mean of 3.6 years (ranging from 1.5 to 5.5 years). The 14 intermediates (mean age $=10.8$ years) had been taking private instruction for a mean of 5.3 years (ranging from 3 to 8 years). Children-were classified as beginners or intermediates according to the difficulty of their musical pieces (see Skill Classification Section below).

\section{Materials and Apparatus}

The children were asked to bring to the laboratory two musical pieces to perform that they had practiced for several weeks (mean time spent practicing the pieces $=7.5$ weeks). The pieces were simple ( 1 to 2 pages); most of the pieces contained a melody and simple harmonic accompaniment, and some of the intermediates' pieces contained additional musical voices. Most of the pieces came from children's training books and song collections (see Appendix).

The children performed the pieces on a computermonitored Yamaha Disklavier acoustic upright piano, which records with optical sensors without affecting the touch or sound of the acoustic instrument. The timing resolution was $2 \mathrm{~ms}$ for note events, with precision (measured by the standard deviation of onset-to-onset durations during recording) within $0.8 \%$ for durations in the range of the performances. The pitch and timing values for each note event were recorded on a Dell $\mathbf{4 8 6}$ computer, which detected all incorrect pitch events by comparing the performances with the ideal values in the musical scores.

\section{Skill Classification}

Because the same children's music is often classified at different levels of difficulty in different training books, a difficulty measure for each piece was collected instead from experts - music teachers and performers. Approximately 100 musical pieces collected from children's performances (including some that were not part of this study; see below) were categorized on the basis of their difficulty level. Four coders (two piano teachers and two pianists) were given copies of the musical scores (the versions from which the children played) and were asked to classify the music into 3 or 4 groups, on the basis of their difficulty (the coders did not hear the performances). The coders chose 4 groups: beginner, intermediate, advanced, and very advanced. The children's performances were then categorized according to the codings of the musical scores, with the constraint that each child's two performances were placed into the same group (in two cases a child's two performances were placed in separate categories by one coder; in this case, the pieces were placed in the beginner category chosen by the remaining coders). Consensus was reached when at least 3 of the 4 coders rated the scores as belonging to the same category. This assignment resulted in coders' consensus on the beginner and intermediate groups, with 15 beginners ( 30 performances) and 14 intermediates (28 performances). The other two groups had insufficient coder agreement 


\section{CORRECT}

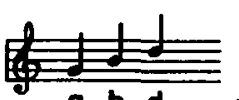

g b d
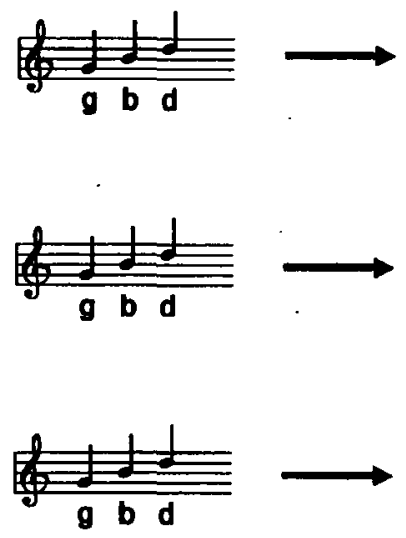

$9 \mathrm{~b} d$
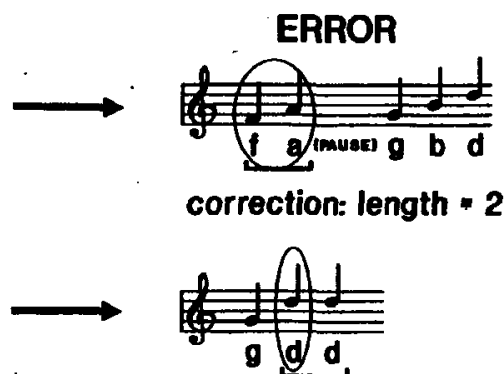

direction: anticipatory distance: +1

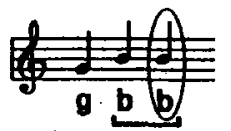

direction: perseveratory distance: -1

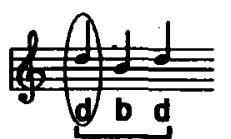

direction: anticipatory

distance: +2
Figure 1. Coding examples for correction and movement errors.

1 (advanced group) or too few cases (very advanced group) and were not included in the study.

\section{Procedure}

Each child and his/her parents were first interviewed about the child's musical background, including years of musical training and experience and weeks of practice on each piece. Then the child was asked to perform one of the pieces, to get acquainted with the piano. A second performance of the same piece was recorded; only the second performance of each piece was included in the analyses. The familiar pieces were performed from the child's notated musical scores. Then the child was asked to indicate his/her musical intentions for the piece, including the melody, phrasing, and dynamics, by pointing to the musical score. The children were able to indicate the melody but only some could indicate phrasing or dynamics. The same procedure was repeated on the next musical piece, after each child participated in an unrelated sightreading task. Each child chose a gift at the end of the session from a collection prominently displayed in the laboratory, and the parents received $\$ 8$ to defray travel expenses.

\section{RESULTS AND CONCLUSIONS}

\section{Pitch Error Coding}

Pitch errors in each performance were identified by computer (each performed pitch was compared with the intended pitches in the musical notation) and coded according to a system similar to those used for coding speech errors (Dell, 1986; Garrett, 1975). Pitch errors can be coded on several dimensions, including size (single note, chord, or note-chord combination), source (contextual / noncontextual), type (addition, deletion, substitution, shift), and movement (anticipatory / perseveratory) (for all possible error types and their codings, see Palmer \& van de Sande, 1993, 1995). Chord errors are defined as multiple pitch errors whose event onsets occurred within a temporal window of $100 \mathrm{~ms}$, based on codings of skilled adults' errors (Palmer \& van de Sande, 1993, 1995). All other errors are identified solely on the basis of pitch (only ordinal temporal information - whether the events were performed in the correct order - was used to identify errors). In addition, all errors were coded for their melodic location (melodic voice, non-melodic voice) and metrical location (beat within measure).

Two types of errors address the monitoring and planning hypotheses: corrections and movement errors (examples are shown in Figure 1). A correction is defined as an error that contains one or more consecutive events played incorrectly, which are followed by a pause and then restarted correctly. A correction's event length is defined as the number of events from the last correctly produced event before the pause up to the pause (in Levelt's (1983) terms for speech errors, the reparandum plus the delay). Corrections can also be coded for their metrical location (between which beats in a measure the interruption occurs).

Movement errors comprise a subset of pitch errors (errors with event lengths equalling one and with no pauses or restarts) that involve two interacting units: a target (intended event) and an intruder (unintended event) whose potential source can be identified from other locations in the musical score. Previous study of performance errors suggested that the coding of an error's movement only when the potential source is in the immediately neighboring musical context (contextual) may be too strict (Palmer \& van de Sande, 1995). Therefore, all errors whose intruder had an identifiable source in the (short) musical scores were coded for direction and distance of movement, analogous to the coding of speech errors (Dell, 1986; Garrett, 1975). The direction of movement from an error to its potential source or nearest same pitch can be forward (anticipatory) or backward (perseveratory). The distance of movement errors can be measured by the number of events from the error to its potential source (positive values = anticipatory, negative values $=$ perseveratory).

This coding resulted in a total of 153 pitch errors and 43 corrections in the beginners' performances, and 298 pitch errors and 55 corrections in the intermediates' performances. Two pianists (beginners) made only 

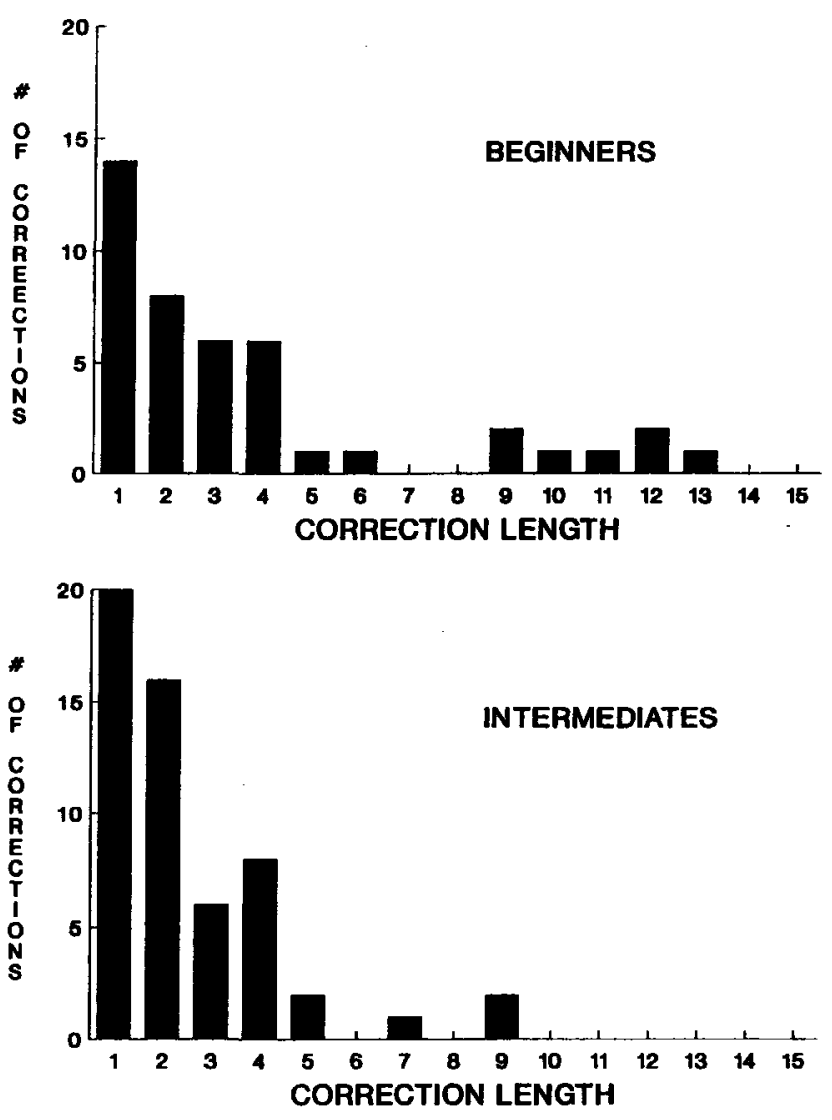

Figure 2. Frequency distribution of correction lengths (in number of events) for beginner and intermediate child pianists.

correction errors; three (one beginner and two intermediates) made only pitch errors; the rest made both correction and pitch errors.

\section{Error rates, age, practice, and musical training}

Each child's error rates were computed relative to the statistical characteristics of the notated music. Pitch error rates were defined as (number of single-note pitch errors / number of total single notes in the notated score) plus (number of chordal (multiple simultaneous event) errors / number of event locations in the notated score). This measure adjusted for both the lengths of musical pieces and the relative densities of single-note and chordal events

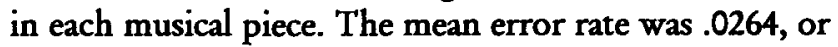
approximately $2-3$ errors out of every 100 pitch events; the range was .0 to .067 . (Because corrections contained a consecutive sequence of related pitch errors, they were analyzed separately and were not included in overall error rates).

Beginner and intermediate groups were first compared on mean error rates, age, amount of training (in years of private piano instruction), and amount of practice reported for each piece (in weeks). Only amount of musical training differed significantly between beginners (mean $=3.6$ years) and intermediates (mean $=5.3$ years); $F(1,27)=7.41, M S_{e}=2.78, p<.05$. Especially important, error rates did not differ between the groups (beginners $=.024$; intermediates $=.029$ ), indicating that the pieces were appropriately difficult for both skill levels. Multiple regression analyses predicting individual performers' error rates from these variables also indicated that training best predicted individual error rates $(r=.37$, $p<.01)$, with no significant improvement when age or practice predictors were added (only age and training predictors were correlated; $r=.54, p<.01$ ). Thus, musical training - not age or practice - was related to both group and individual performance.

Error rates may reflect a speed-accuracy tradeoff, such that performers who play at a faster tempo make more errors. To test this possibility, the error rates were correlated with the tempo of each performance across individuals. Performance tempo was measured by the average beat duration in the first error-free measure in each performance ${ }^{1}$. The beat level, as determined by the denominator of the notated time signature for each piece, was the quarter-note for 56 of 58 pieces. The mean beat duration was $548 \mathrm{~ms}$ (109 beats $/ \mathrm{min}$ ) and there were no tempo differences across groups (beginners' mean beat duration $=565 \mathrm{~ms}$; intermediates' $=529 \mathrm{~ms}$ ). The correlation between error rates and beat duration was negative but not significant $(r=-.25, p>.05)$, indicating that error rates were not simply a function of speed.

\section{Monitoring}

Corrections. The monitoring hypothesis predicts that skilled performers have greater ability to monitor and correct their performances. There was no difference in the number or rate (relative to the length of the pieces) of correction errors for the two groups. There was, however, a difference in how quickly performers identified and corrected errors; the length of each correction is shown in Figure 2 for beginners and intermediates. The mean correction length was longer for beginner pianists than for intermediates (analyses by subjects; $F(1,24)=3.1$, $M S_{e}=5.55, p=.08$; analyses treating performances as independent: $\left.F(1,96)=4.86, M S_{e}=7.24, p<.05\right)$. Mean lengths were 3.70 events for beginners and 2.5 events for intermediates, supporting the hypothesis that monitoring improves with attained skill ${ }^{2}$.

1 It was not possible to base tempo estimates on larger sections of the children's performances because of frequent correction errors, which contain pauses that would confound tempo estimates.

2 If correction lengths reflect instead an unwillingness to correct mistakes, which increases with skill level, then intermediates' performances should contain fewer correction errors or a higher proportion of runs (neighboring uncorrected errors) among pitch 


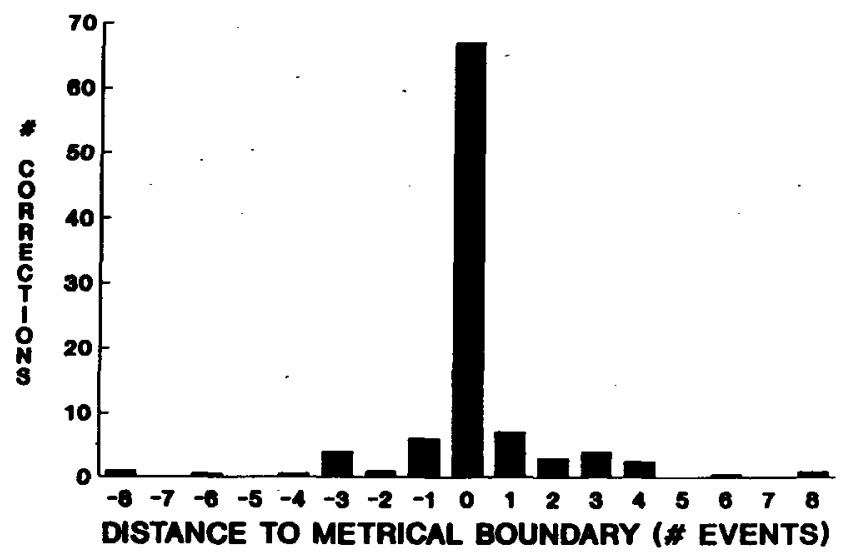

Figure 3. Frequency distribution of distance (in number of events) between correction locations and nearest metrical boundary for all child pianists.

Detection near constituent boundaries. The monitoring hypothesis also predicts that error detection may be enhanced near constituent boundaries (Levelt, 1983). Metrical barlines, prominently indicated in musical notation by vertical lines, provide visual markers for metrical unit (measure) boundaries; the event following the barline is often perceived as having the strongest metrical accent. The distance between the location of each correction and the nearest metrical barline is shown in Figure 3. The majority of corrections (68\%) occurred at metrical barlines (between the last event in one measure and the first event in the next measure). There were no differences between beginners and intermediates, suggesting that detection is most sensitive for both groups near metrical boundaries. Pitch errors were not more likely to occur at metrical boundaries for either group $(p>.05)$; thus, the increased occurrence of corrections at metrical boundaries was not simply a product of stimulus characteristics such as number of pitch events intended to be produced at those locations.

Corrections that occur at metrical boundaries may reflect performers' tendency to delay interruption until after completion of the current metrical unit, instead of a failure to detect errors according to the monitoring hypothesis (Levelt, 1983). That is, performers may complete certain musical units before interrupting. This delay explanation would predict that a higher percentage of delayed interruptions (corrections with lengths greater than one event) than of immediate interruptions (corrections with lengths equal to one event) should occur at metrical boundaries. We compared the tendency of long and short corrections to respect metrical boundaries. $68 \%$

errors than those of beginners. In contrast, intermediates had more corrections and no higher proportions of pitch error runs than beginners. of long corrections and $72 \%$ of short corrections occurred at metrical boundaries; there were no differences between beginner and intermediate groups. Thus, the lack of difference in metrical location between long and short corrections suggests that the tendency of corrections to coincide with metrical boundaries is due to enhanced error detection abilities, rather than a tendency to delay interruption until completion of a metrical unit.

\section{Planning}

Direction of errors. The planning hypothesis predicts more anticipatory and fewer perseveratory errors for higher skill levels (as a consequence of more future-oriented planning). The monitoring hypothesis predicts that skilled performers may be able to monitor against perseverations of events that have already occurred, but makes no predictions for change in anticipations (events that have not yet occurred). The direction of contextual movement errors (errors in which an intruder has an identifiable source in the immediately surrounding musical context) was computed for additions, because only they differentiate predictions of the monitoring and planning hypotheses (for example, perseveratory substitutions, in which an unintended event from earlier in the sequence replaces an intended event, may result from either a failure to monitor the event as previously performed or a failure to plan).

An analysis of variance (ANOVA) on the number of errors by movement direction (anticipatory and perseveratory) and skill level (beginners and intermediates) indicated a significant effect of direction, $F(1,25)=10.44$, $M S_{e}=0.64, p<.01$, with more anticipations than perseverations. There was a significant interaction of direction with skill level, $F(1,25)=4.4, M S_{c}=0.64$, $p<.05$; there were more anticipatory (A) and fewer perseveratory $(\mathrm{P})$ errors for intermediates (anticipatory proportion or $\mathrm{A} /(\mathrm{A}+\mathrm{P})=.73)$ than for beginners $(A /(A+P)=.57)$, supporting the hypothesis of increased future-oriented planning with skill acquisition.

Because the number of errors is partially determined by the stimulus characteristics (number of opportunities for errors), which differed across performers' pieces, the direction of movement was also examined in terms of the relative proportion of each performer's total errors. Figure 4 shows the proportion of anticipatory and perseveratory addition errors for both groups. An ANOVA indicated higher proportions of anticipatory than perseveratory errors for both groups, $F(1,25)=10.12, M S_{e}=.006$, $p<.01$. Although the interaction of direction with skill level was not significant for the error proportions, the intermediates tended to show less perseveratory behavior than the beginners.

Distance of errors. The planning hypothesis predicts that skilled performers incorporate larger ranges of events 


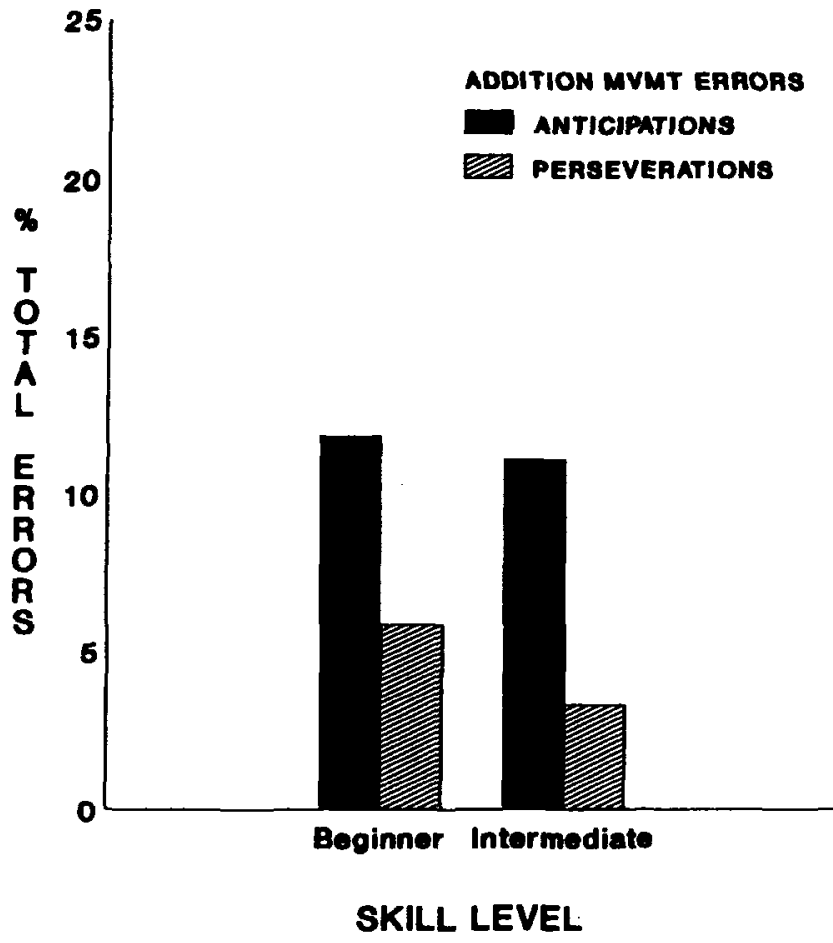

Figure 4. Proportion of anticipatory and perseveratory addition errors for beginner and intermediate child pianists.

(both perseveratory and anticipatory) in mental plans as a result of greater accessibility of sequence events, and thus interacting elements in errors may span larger distances. Figure 5 shows the frequency distribution of distances spanned by all movement errors (additions, substitutions, and shifts), in number of events between an intruder and its nearest possible source in the musical score, for the two groups. Only errors whose distances fell within the length of the shortest piece ( 30 events) were included, to adjust for the lengths of different musical pieces. The standard deviation, a measure of range, was significantly larger for the intermediates ( $s d=8.72$ events) than for the beginners ( $s d=6.39$ events) (Bartlett's test of homogeneity of variance; chi-squared (1) approximation $=10.66, p<.01$ ), supporting the hypothesis of increased range of planning with skill acquisition.

Some pitch errors may not necessarily arise from a source in the musical sequence, despite the presence of that pitch somewhere in the musical score. Therefore, the analysis was repeated to include only errors whose distances fell within one measure on either side of the error. Rate of pitch repetitions in the musical pieces may also influence the distance measures; pieces with higher pitch repetition rates should have smaller distances on average. To adjust for this possibility, the analysis was repeated to include only errors whose distances fell outside the computed mean distance between any given
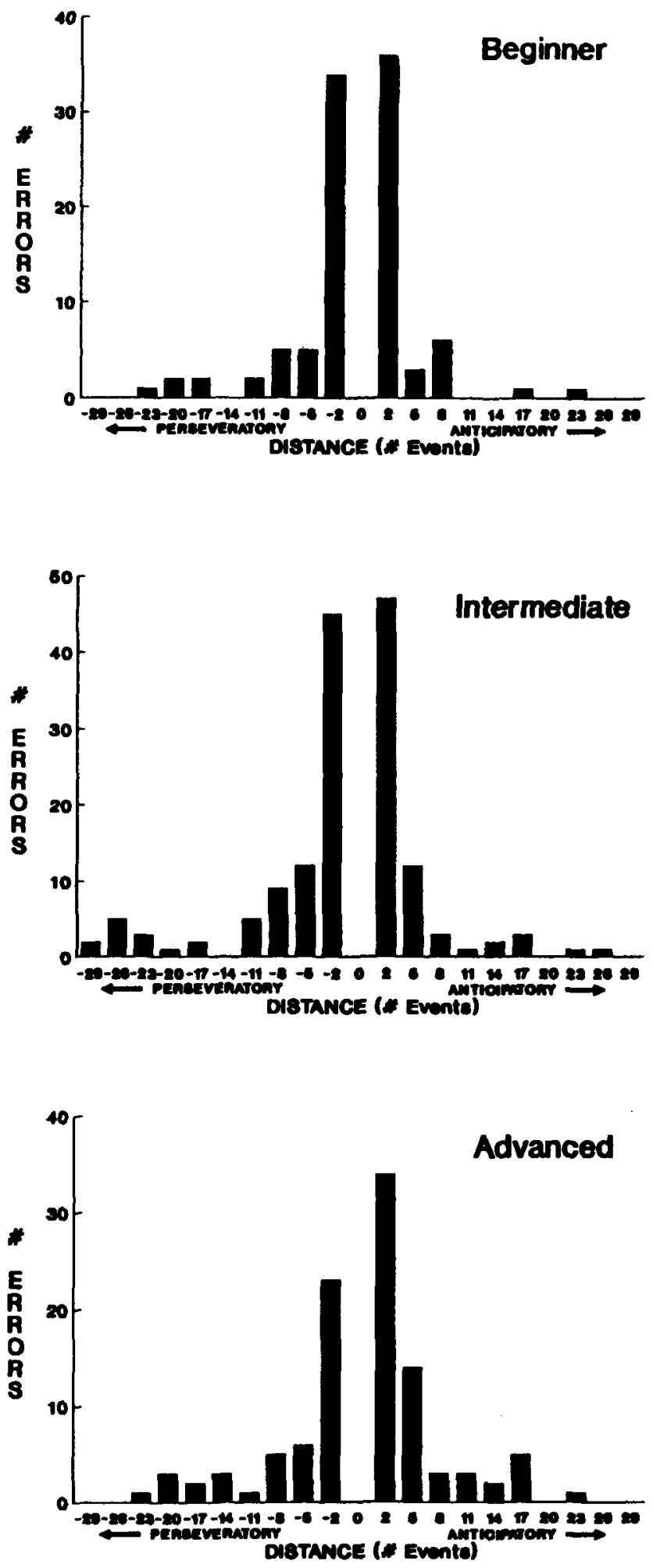

Figure 5. Frequency distribution of distance (in number of events) between all movement errors and their nearest same-pitch sources in the musical score for beginner, intermediate, and adult pianists. (With the exception of the zero-point, abscissa values denote the midpoint of each bin.) 


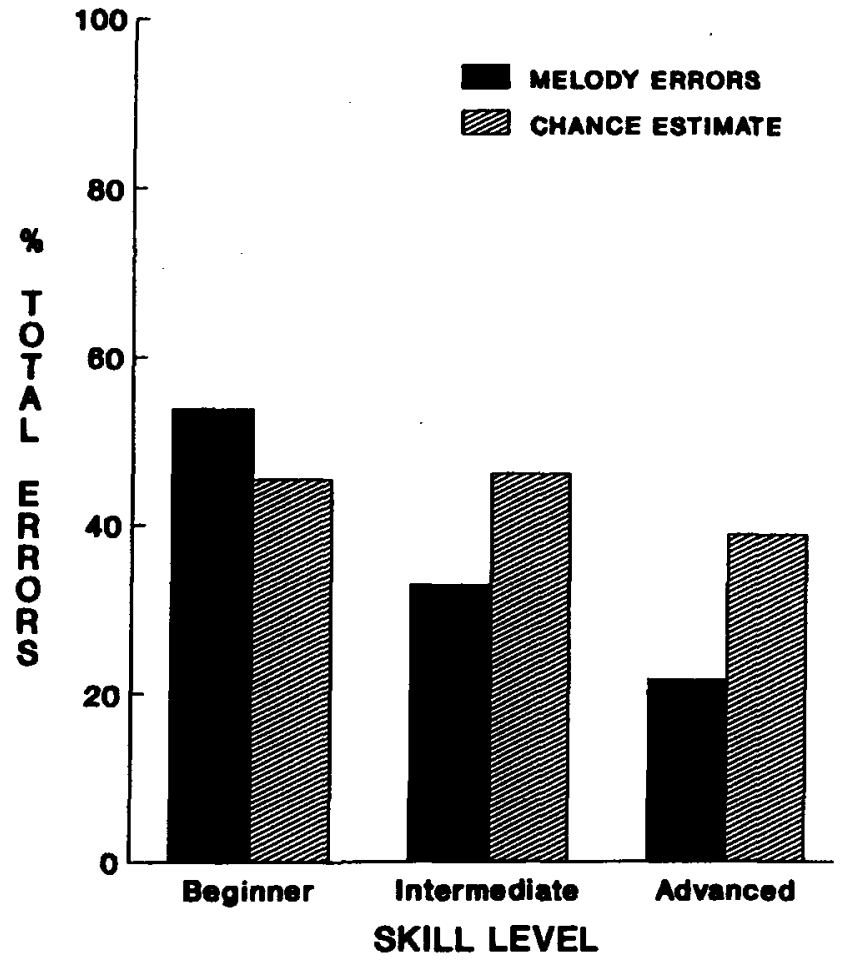

Figure 6. Percentage of total errors occurring in the voice chosen as melody for beginner, intermediate, and adult pianists, and chance estimates (based on proportion of melodic events relative to total events in the score).

pitch event and its reoccurrence in the musical notation (which yielded a mean chance estimate of recurring pitch events every 11.23 events). These conservative analyses yielded the same findings of larger standard deviations for intermediates than beginners. Thus, interacting elements in movement errors tended to span larger numbers of events for intermediates than for beginners, regardless of the window of events included in the analysis.

Distance measures based on number of intervening events do not take into account the temporal structure of the musical events, in particular, the amount of elapsed time. For example, the same number of events may encompass a larger temporal span in one performance and a smaller temporal span in another. Error distances were recomputed in absolute time units (ms) by multiplying the number of events between each error's intruder and its source in the musical score by the mean event duration (based on the mean beat duration divided by the number of events per beat) for each performance. Error distances based on this temporal measure did not differ across skill levels; intermediates' range ( $s d=1917 \mathrm{~ms}$ ) was similar to beginners' $(s d=2083 \mathrm{~ms}$ ). Thus, the temporal range of planning was similar across skill levels.
Sensitivity to structural relationships.

Melodic structure. We pursued children's sensitivity to structural relationships first by examining influences of melodic structure. The melodic voice was determined from each pianist's verbalized interpretations, which were then marked on the musical score by the experimenter. Figure 6 shows the proportion of errors occurring in the voice chosen as melody by each child. Because each musical piece contains different percentages of melodic events, analyses are based on percentage, rather than number, of total errors. An ANOVA on each pianist's proportion of errors in the melody showed a significant difference across groups, $F(1,24)=4.2, M S_{e}=.06, p<.05$, with higher proportions for beginners than intermediates. The probability of an error occurring in the melody by chance, estimated by the proportion of melodic events relative to total notated events, was equivalent for the two groups (beginners' pieces: 45.4\%; intermediates' pieces: $46.1 \%)$. Thus, the lower proportion of errors in the melody by the intermediate group was not simply a result of increased number of nonmelodic voices and events in the more difficult musical pieces. The proportion of errors in the melody differed significantly from the chance estimate only for intermediates, $t(13)=2.7, p<.05$. These results suggest that musical events become less prone to error with skill acquisition.

Repeating pitch events. Increased sensitivity to structurally important events may also be manifested by decreased sensitivity and thus increased errors for less important events. Repeating pitch events in music often are ordered such that successive (neighboring) instances are less important structurally than the first instance, and hence may be more prone to error if they are perceived as less significant. $68 \%$ of errors on repeating pitches (most often deletions) for the two groups were errors of the second instance of two identical (repeating) pitches; there were no group differences. (Cases in which more than one repetition of the same pitch occurred were coded as successive repeating pitches.)

Figure 7 shows the percentage of errors involving successive repeating pitches and the mean chance estimates (based on percentage of pitches in each piece that repeated) for each group. Intermediates had significantly higher proportions of repeating pitch errors than beginners, $F(1$, 25) $=4.5, M S_{e}=.016, p<.05$. As shown in Figure 7, the chance estimates (proportion of total pitches that involved repeating events) were similar for intermediates' pieces (mean $=15.3 \%)$ and beginners' (mean $=16.7 \%)$. Only the beginners' repeating errors differed from the chance estimate, $t(12)=-4.003, p<.01$, with fewer repeating pitch errors than chance. Thus, intermediate pianists were less sensitive to repeating pitches than beginners. 


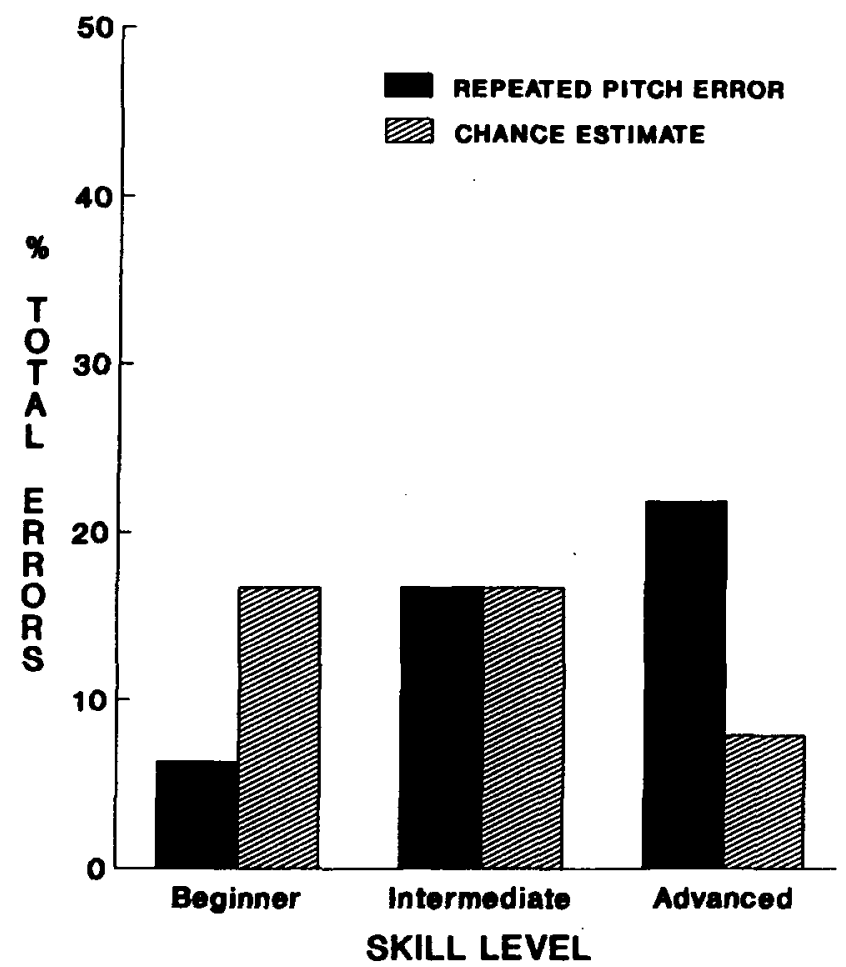

Figure 7. Percentage of total errors occurring on successive repeated pitches for beginner, intermediate, and adult pianists, and chance estimates (based on proportion of successive repeated pitches relative to total events in the musical score).

In summary, pianists of intermediate skill levels showed greater error detection abilities, more anticipatory behavior, larger ranges of planning, greater sensitivity to melodic structure, and decreased sensitivity to repeating pitch structures, than beginner musicians. The skill level differences were not due to increased difficulty of the music; overall error rates remained the same across beginners and intermediates. Instead, these findings suggest that planning and monitoring abilities underlie cognitive changes during early years of musical skill acquisition. Both groups demonstrated sensitivity to metrical boundaries, more anticipatory than perseveratory behavior, and overall performance levels (measured by error rates) corresponding to amount of musical training.

\section{Experiment 2: Advanced Skill Levels}

Children's music performances revealed increased capacities for monitoring and adjusting to one's own performance, and maintaining information from larger ranges of material during planning. These influences reflect the availability of knowledge about events other than those currently being produced; presumably these behaviors would be more prevalent in advanced skill levels. In addition, children with more musical training demonstrated increased sensitivity to musical structure. If monitoring and planning capacities rely on increased memory associations among musical events and structures, then errors in skilled adult musicians' performances should exhibit stronger biases toward structurally important events than children's errors as a result of the adults' cumulative exposure to and practice with the same forms of Western tonal music. We test this hypothesis by examining error patterns in skilled adult pianists' performances of well-learned music.

Although the task classification method for categorizing children's musical pieces in the previous experiment was useful for identifying beginner and intermediate skill levels, it did not aid in identifying children of advanced skill levels. In addition, it is difficult to find a large number of child pianists of very advanced levels. Therefore, an adult population of skilled pianists was sought out for comparison. The pianists were chosen from a homogeneous group of students in a doctoral training program in piano performance. Instead of performing on the upright piano that the children played, the adults performed on an acoustic computer-monitored grand piano, similar to the types of piano on which they were used to practicing.

In addition to performing two musical pieces which they had practiced for several weeks, the adult pianists indicated detailed conceptual interpretations of the pieces, by marking them on the musical notation. These interpretations, which included indications of the melody (primary voice), phrase structure, dynamics, and other conceptual intentions, were then used to test hypotheses concerning sensitivity to musical structure. Adults' error patterns should reflect heightened sensitivity to musical structure relative to children's performances, including fewer errors in the voice interpreted as melody and more repeating pitch errors. We also expect that adults should exhibit larger percentages of errors that reflect advanced monitoring and planning capacities, including shorter correction lengths and greater ranges of planning. The adults' error patterns were compared with those of the beginners and intermediates, to see if performers exhibit the greatest change in monitoring and planning capacities relatively early (from beginner to intermediate levels) or late (to advanced levels) during skill acquisition.

\section{METHOD}

Participants

10 adult pianists (mean age $=27$ years) were recruited from the Doctor of Musical Arts degree program at the Ohio State University School of Music. The pianists had been taking private instruction for a mean of 18 years (range $=12$ to 25 years).

\section{Materials and Apparatus}

The adults were asked to bring two musical pieces to 
perform that they had practiced for several weeks (mean time spent practicing the pieces $=15$ weeks). The pieces were complex; most of the pieces contained 3 to 5 voices, many exhibited particular musical forms, such as sonata form, and many contained ornaments and embellishments. Because the adults' pieces tended to be much longer than the children's pieces, only the first large section (2-3 pages) of each musical piece was included for analysis (the adults performed the pieces in their entirety). The musical pieces were in 17th - 20th century Western tonal styles (see Appendix).

The adults performed the pieces on a computer-monitored Boesendorfer SE acoustic concert grand piano, which records with optical sensors without affecting the touch or sound of the acoustic instrument. The timing resolution was $1.25 \mathrm{~ms}$ for note events, with precision (measured by the standard deviation of onset-to-onset durations during recording) within $0.3 \%$ for durations in the range of the performances. The pitch and timing values for each note event were recorded on the computer, which detected all incorrect pitch events by comparison with the ideal values in the musical scores.

\section{Procedure}

The adults were first interviewed about their musical background, including amount of musical training and experience, and specific practice on each piece. Then they were asked to perform one of the pieces and a recording was made. A second performance of the same piece was recorded; only the second performance of each piece was included in the analyses. The adults were then asked to notate on the musical score their interpretations of melody (most important voice), phrasing, dynamics, and any other relevant information; they were able to notate all of these aspects. The adults performed the pieces from the notated musical scores. The same procedure was repeated on the next musical piece, after the adults performed an unrelated sight-reading task. The adults were paid $\$ 8$ at the end of the session for their participation.

\section{RESULTS AND DISCUSSION}

\section{Pitch Error Coding}

Pitch errors in the adults' performances were identified and coded in the same manner as were the children's errors. Events comprising musical ornaments, such as trills and appoggiatura, were not included because their specifications in the musical score allow some flexibility in how they are performed. There was a total of 2 correction errors and 280 pitch errors in the adults' performances. Only two pianists made correction errors; all of the pianists made pitch errors.

\section{Error rates, age, practice, and musical training}

Adults' overall error rates were computed the same way as for the children's performances. Individual error rates ranged from .004 to .13; the mean error rate was .0264 . The adults' error rates did not differ from those of the beginner or intermediate groups, indicating that the pieces were appropriately difficult for all groups.

Error rates were examined next in terms of age, musical training (number of years of private instruction), and practice reported for each musical piece (number of weeks). Multiple regression analyses predicting individual performers' error rates from each combination of these variables indicated that practice was most strongly related to error rates $(r=-.44, p<.01)$, with no significant improvement in the fit when age or training predictors were added (only age and training predictors were correlated; $r=.62, p<.01$ ). Thus, amount of practice - not training, as seen for the children - predicted individual skill levels in adult performances.

To test whether adults' performances reflect a speedaccuracy tradeoff, the error rates were correlated with the tempo of each performance. Performance tempo was measured by the mean quarter-note (beat) duration, based on the first error-free measure duration in each performance. The beat level was determined by the denominator of the notated time signature for each piece; the beat level was the quarter-note for 16 of 20 pieces. The mean quarter-note duration was $843 \mathrm{~ms}$ (or 71 beats/min) and there was a wide range of tempi (318 to $1587 \mathrm{~ms} / \mathrm{beat}$ ); the adults' pieces were performed at a slower tempo on average than the children's pieces, $F(2,36)=10.5$, $M S_{e}=31361.6, p<.01$. The correlation between error rates and mean beat duration was small and positive $(r=.12, p>.05)$, indicating that error rates were not a function of speed.

\section{Monitoring}

There were very few correction errors $(n=2)$ in the adults' performances, consistent with advanced monitoring abilities at high skill levels, which prevented analyses of correction lengths or monitoring abilities near constituent boundaries.

\section{Planning}

Direction of errors. The direction of contextual addition movement errors (errors in which an added intruder has an identifiable source in the immediately surrounding musical context) was examined next. Although the proportion of anticipatory errors relative to total errors (mean $=7.8 \%$ ) was approximately twice that of perseveratory errors (mean $=4.2 \%$ ), there were too few in number $(n=23)$ to allow comparison with the childrens' errors. The scope of movement errors was therefore extended in a subsequent analysis to include those addition errors whose intruders came from larger distances in the musical score (distances up to 30 events, the length of the 
shortest children's piece, were included). There were significantly greater proportions of anticipations than perseverations (sign test by pianist, $p<.05$ ), consistent with the children's error patterns.

Distance of errors. The distances spanned by all movement errors (additions, substitutions, and shifts) were computed for the adults' performances, in number of events between an intruder and its nearest source in the musical score. This measure of range of planning was adjusted for comparison with the childrens' performances by including in the analysis only errors whose distances fell within the length of the shortest musical piece ( 30 events), and is shown in Figure 5. The standard deviation of the intruder-to-source distances was $\mathbf{8 . 2}$ events (approximately one measure); this was significantly larger than the beginners' (= 6.40 events) distance measures (Bartlett's test, chi-squared approximation $(1)=2.83$, one-tailed $p<.05$ ) but not the intermediates' ( $=8.72$ events).

Distance measures were recomputed in absolute durations to take into account the temporal structure of the musical events. As with the children's errors, the intruder-to-source distances were computed in ms by multiplying the number of event durations by the mean event duration for each performance. The standard deviation for adults' errors (in ms) was larger ( $s d=2499$ $\mathrm{ms}$ ) than that of both the beginners (= $2083 \mathrm{~ms}$; Bartlett's test, chi-squared $(1)=3.29, p<.05)$ and the intermediates (= $1917 \mathrm{~ms}$; Bartlett's test, chi-squared $(1)=8.91$, $p<.05)$. The increased range of planning from children to adults, measured in absolute time units, was not simply a function of tempo; the correlation of tempo with standard deviations within individuals was not significant across groups $(r=.14, p>.05)$ or within groups (beginners: $r=.37, p>.05$; intermediates: $r=.26, p>.05$; adults: $r=.33, p>.05$ ). Thus, distance measures based on the same number of intervening events reflected larger amounts of elapsed time for the adult performances than for the beginners' and intermediates' performances.

\section{Sensitivity to Musical Structure}

Melodic structure. We pursued adults' sensitivity to structural relationships first by examining influences of melodic structure. The melodic voice was determined from each pianist's notated interpretations of melody (primary voice). As shown in Figure 6, the mean percentage of errors in the melody for adult pianists was lower than the mean proportion of total events that occurred in the melody in each piece, $t(19)=3.73, p<.01$.

Sensitivity to melodic structure was compared next across skill levels. An ANOVA on each pianist's percentage of errors in the melody indicated significant differences among the three groups, $F(2,33)=5.45, M S_{\mathrm{e}}=.055$, $p<.01$. Post-hoc comparisons indicated that adults had a significantly lower proportion of errors in the melody (21.6\%) than beginners (54\%; Dunn-Bonferroni, $p<.05$ ) and a lower (non-significant) proportion than intermediates (33\%). As shown in Figure 6, the chance estimates were similar for the three groups; the difference between groups was not simply a result of increased proportions of nonmelodic events in the more difficult musical pieces. These results suggest that the melody becomes less prone to error with increased skill as it becomes conceptually prominent.

Repeating pitch events. Finally, we examined adult pianists' sensitivity to repeating pitch events. $85 \%$ of successive repeating events that were in error (most often deletions) were errors of the second instance of the repeating event. The repeating pitch errors occurred significantly more often than expected by chance, $t(9)=3.72, p<.01$, based on the percentage of total pitch events in each piece that repeated. Thus, adult pianists were less sensitive to (made more errors for) repeating pitches than expected.

The adults' performances were next compared with those of the children; as shown in Figure 7, the adults made significantly higher proportions of repeating pitch errors than the children, $F(2,34)=4.7, M S_{e}=.016$, $p<.05$, despite the higher proportions of repeating pitches in children's piece $(16 \%)$ than in adults' pieces (7.9\%); the increase in repeating pitch errors between groups was not a result of differences in the musical pieces. Post-hoc comparisons indicated that the adults had significantly higher proportions of repeating pitch errors than beginners (Dunn-Bonferroni, $p<.05$ ) but not intermediates. More importantly, only adult pianists not beginners or intermediates - exhibited less sensitivity to repeating pitches than expected by chance $(p<.05)$, as shown in Figure 7.

To test whether decreased sensitivity to repeated pitch structures is accompanied by increased sensitivity to melody, the two error types were correlated across individuals. First, difference scores were computed for each individual between each of the two error types and the expected proportion (chance estimates) from their pieces. These difference scores (melody error proportion minus chance estimate, repeated pitch error proportion minus chance estimate) were negatively correlated across the three skill levels $(r=-.56, p<.01)$. As performers gained sensitivity to melodic structure, they showed decreased sensitivity to repeated pitch structure.

In summary, skilled adult performances displayed greater temporal ranges of planning, increased sensitivity to melodic structure, and decreased sensitivity to repeating pitches. Evidence for both planning and structural sensitivity in adults' performances significantly increased from beginners' performances, and slightly increased from intermediates' performances. 


\section{General Discussion}

Differences of two to three years of musical training revealed increased cognitive capacities underlying children's ability to perform music: monitoring and adjusting one's own performance, maintaining information during planning from large ranges of the musical sequence, and heightened sensitivity to musical structure. Monitoring skills were reflected in increased speed of error detection and correction by intermediate pianists, and higher detection sensitivity at metrical constituent boundaries by both beginners and intermediates. Children's corrections reflected delays in error detection, not delays in interruption, similar to findings in speech monitoring (Levelt, 1983). Planning skills were reflected in influences of events from larger ranges of material for intermediate than beginner pianists, as well as more anticipatory than perseveratory behaviour. Advanced adults' performances demonstrated increased temporal range of planning relative to beginners and intermediates. Finally, sensitivity to melodic structure increased and sensitivity to repetitive pitch structure decreased across skill levels. These findings extend effects that have been documented for highly skilled behaviour in many domains, such as music, speech, and typing, to much earlier stages of skill acquisition. Increased access to knowledge about events other than those currently being produced (Garrett, 1975; Lashley, 1951; Rumelhart \& Norman, 1982; Shaffer, 1976).

The abilities to monitor and to plan may both rely on increased access to relevant information from memory during performance. According to monitoring explanations, skilled performers detect and correct deviations from their intentions; when trouble is detected, corrective action is taken, based on internal information (intentions) to which the performer has access, as well as external information (Levelt, 1989). The increased ability of advanced musicians to state their conceptual interpretations of music suggests that musical intentions are strengthened with skill. According to planning explanations, skilled performers' complex plans incorporate both future-oriented (anticipatory) and past-oriented (perseveratory) events from larger ranges of a sequence (Palmer \& van de Sande, 1995). Anticipatory and perseveratory errors can arise in associative models of planning in sentence production from activation rising more quickly for those elements than for current intended (correct) elements, due to priming from other related events or nodes (Dell, 1986; MacKay, 1987). A bias toward anticipatory behaviour can arise from practice; Dell, Berger, and Svec (1997) proposed a model of sentence production in which practice affects the strength of connections in mental plans to both present and future events but not to past events. Thus, monitoring and planning capacities both improve with skill as a function of increased memory associations among elements of musical knowledge.

Sensitivity to melodic structure continued to increase across skill levels, evidenced by decreasing error rates for events in the musical voice conceptualized by performers as melody. The fact that melody gains conceptual prominence by intermediate levels of skill acquisition is not surprising; melody is one of the primary forms of distinguishing structure in multi-voiced music, and is a primary focus of both psychological studies and music-theoretic approaches to musical behaviour (Dowling, 1978; Lerdahl \& Jackendoff, 1983; Narmour, 1990). Melodic prominence has been demonstrated in music perception and performance tasks, including listeners' detection of pitch changes in melodic tones (Palmer \& Holleran, 1994), performers' expressive emphasis of the voice interpreted as melody (Palmer, 1989, 1996), and lowered error rates in melodic voices (Palmer \& van de Sande, 1993, 1995; Repp, 1996). Melody is often ambiguous, like many other forms of musical structure; the conceptual prominence of melody in performance may be necessary for the successful communication to listeners of events' relative importance.

One form of musical structure to which performers of all skill levels were sensitive was meter. The majority of beginners' and intermediates' corrections tended to occur at the beginnings of metrical units, similar to findings in speech of increased monitoring abilities near structural (clause) boundaries (Levelt, 1983). Metrical barlines, notated in musical scores by prominent vertical lines between musical events, provide an obvious visual marker in musical scores that demarcate equivalent time-spans. Other forms of musical structure, such as melody, have no explicit markers in musical notation, and must be learned from other sources.

Advanced skill levels displayed decreased sensitivity to less important events, in particular, to repeating pitches. Adults' advanced performances exhibited more repetition failures than children's, despite the fact that the simpler pieces of children's musical repertoire tend to contain more repetition than the adults' advanced musical repertoire. Repetition failures in music performance resemble repetition deafness effects documented in language comprehension and production. Explanations of repetition deafness in language production often rest on internal monitoring mechanisms, responsible for detecting and deleting error-like patterns such as item repetitions, that occasionally fail (Shattuck-Hufnagel, 1979). An explanation from node structure theory proposes that encoding proceeds by forming many-to-one connections in memory between lexical nodes (representing lexical concepts) and phrase-level nodes (MacKay, 1987). Repetition deafness results because the many-to-one connections from repeating lexical concepts to phrase nodes must be formed sequentially and are more prone to error under time pressure (Miller \& MacKay, 1994). 
In contrast to these explanations of repetition deafness, we propose that advanced musical skill levels increasingly differentiate the conceptual strength of musical events; conceptual structure accounts for both melodic sensitivity and repetition deafness. This interpretation is supported by the negative association found between performers' melodic error rates and repeated pitch error rates. Beginning musicians are less able than advanced musicians to distinguish structurally important from less important events, evidenced in part by their less detailed interpretations. The greater the conceptual weighting of a given item, the less prone it is to error during retrieval. Therefore, children's failures to weight melody as conceptually strong or repeating pitches as conceptually weak result in different error patterns than those of adults.

Increased sensitivity to structural relationships during the acquisition of musical skill is not surprising; one of the goals of musical training is to heighten performers' awareness of different musical structures, and melody and meter are primary forms of musical structure. What is surprising is that the effects of musical training on cognitive capacities can be quantified so early in skill acquisition (in the first 3-5 years), rather than after many years of deliberate practice typical of skilled musicians. Diary studies of music performance emphasize the role of accumulated practice in accounting for skill differences (Ericsson, Krampe, \& Tesch-Romer, 1993; Sloboda, Davidson, Howe, \& Moore, 1996); accumulated practice would predict larger differences among the adult and children performances reported here than among the beginner and intermediate performances, which differed only in a few years of musical training and experience. In contrast, the largest differences in monitoring and planning abilities were documented between beginners and intermediates, with adults showing significant increase beyond childrens' performances only in heightened sensitivity to musical structure. Thus, changes in error types across skill levels suggest that the cognitive bases of planning and monitoring change most at early stages of skill acquisition, whereas sensitivity to structural features, which are particular to a given musical piece or performance, is refined in later stages.

Practice and training variables differed in predicting overall error rates as well among skill levels. Amount of musical training - not age or specific practice on a musical piece - differentiated the children's overall performance levels, both on an individual and group basis. In contrast, adults' overall performance was best predicted from specific practice. Diary-based studies of musicians' practice habits indicate that accumulated hours of practice (not age or musical experience) best differentiate among skill levels (Ericsson et al., 1993; Slobodaet al., 1996). Although these studies do not contrast practice with performance abilities on specific musical pieces, a comparison with the current findings suggests that when practice and training issues can be separated they play different roles at different stages of skill acquisition. Early in skill acquisition, pianists must acquire specific cognitive skills essential to music performance; in particular, the planning and monitoring skills required for competent (correct) performance, which are prerequisite for advanced skill levels. Improvements by advanced adult performers are evidenced in more subtle aspects, such as the measures of sensitivity to musical structure reported here. The amount of specific practice on a particular piece is less important to the acquisition of basic cognitive capacities underlying music performance than to the honing of particular (already competent) performances. Thus, amount of training may be more indicative of overall performance differences at earlier stages of skill acquisition and amount of practice on a particular piece indicative of performance differences at advanced skill levels.

Although a naturalistic error corpus such as this one offers advantages in generalizing to real-world behavior, it also introduces limitations. One limitation of this study stems from differences in stimulus characteristics across skill levels. The musical repertoire performed by the beginner and intermediate children is similar on many dimensions; however, the music performed by skilled (adult) pianists varies greatly in terms of compositional form and style of music. Furthermore, skilled performers tend to be more well-versed in some types of music than others, whereas novice musicians do not tend to display large familiarity differences. Thus, the chance estimates with which error patterns are compared may not be functionally equivalent across childrens' and adults' performances. In addition, adults' more complex pieces tended to be performed at slower tempi than childrens' performances; adults' temporal range of planning was also larger than that of the children. Although the temporal range of planning was not correlated with performance tempo, the two may reflect underlying stimulus differences. Experimental studies in which the same musical pieces are performed by all musicians would allow better control of stimulus characteristics. The problem arises in finding musical stimulus materials that are appropriately difficult and yield equivalent error rates across all skill levels, a primary advantage of the naturalistic corpus of well-learned performances reported here.

Finally, we report a methodology here that attempts to control for task difficulty in comparisons across skill levels. Most studies of skill differences compare performance on the same task by different participants, for whom the task may not be equally easy. Our method allowed performers themselves to select the stimulus materials appropriate for their skill level, which were sorted on the basis of an objective measure of task difficulty from experts. This method allowed the study of 
performances at different skill levels that differed only in qualitative (type of error), not quantitative changes (amount of error). Without such controls, performance breakdowns from different skill levels may reflect primarily task-related differences, rather than monitoring and planning capacities that contribute to the cognitive bases of skill acquisition.

This research was supported in part by NIMH Grant 1R29-45764 to the first author and by Grant SES-9022192 from the National Science Foundation. This work was conducted while the first author was a Fellow at the Center for Advanced Study in the Behavioral Sciences, Stanford, CA. We are grateful for comments on an earlier draft from Julie Boland, Eric Clarke, John Kihlstrom, In Jae Myung, and Bruno Repp, and for the assistance of Adrianne Blair, Kory Klein, Richard Reach, Grant Rich, and Brent Stansfield. Correspondence may be sent to Caroline Palmer, Psychology Department, Ohio State University, 1885 Neil Ave., Columbus, OH 43210, or to palmer.1@osu.edu.

\section{References}

Bamberger, J. (1986). Cognitive issues in the development of musically gifted children. In R. Sternberg \& J. Davidson (Eds.), Conceptions of giftedness. Cambridge: Cambridge University Press.

Boomer, D.S. (1965). Hesitation and grammatical encoding. Language and Speech, 8, 148-158.

Dell, G.S. (1986). A spreading-activation theory of retrieval in sentence production. Psychological Review, 93, 283-321.

Dell, G.S., Berger, L.K., \& Svec, W.R. (1997). Language production and serial order: A functional analysis and a model. Psychological Review, 104, 123-147.

Dowling, W.J. (1978). Scale and contour: Two components of a theory of memory for melody. Psychological Review, 85, 341-354.

Drake, C., Dowling, W.J., \& Palmer, C. (1991). Accent structures in the reproduction of simple tunes by children and adult pianists. Music Perception, 10, 343-378.

Ericsson, K.A., Krampe, R.T., \& Tesch-Romer, C. (1993). The role of deliberate practice in the acquisition of expert performance. Psychological Review, 100, 363-406.

Garcia-Albea, J.E., del Viso, S. \& Igoa, J.M. (1989). Movement errors and levels of processing in sentence production. Journal of Psycholinguistic Research, 18, 145-161.

Garrett, M.F. (1975). The analysis of sentence production. In G.H. Bower (Ed), The psychology of learning and motivation (pp.133-177). New York: Academic Press.

Garrett, M.F. (1980). Levels of processing in sentence production. In B. Butterworth (Ed.), Language production, vol. 1: Speech and talk (pp.177-220). London: Academic Press.

Gruson, L.M. (1988). Rehearsal skill and musical competence: does practice make perfect? In J.A. Sloboda (Ed.), Generative processes in music: The psychology of performance, improvization, and composition (pp. 91-112). New York:
Oxford University Press.

Henderson, M.T. (1936). Rythmic organization in artistic piano performance. In C.E. Seashore (Ed.), Objective analysis of musical performance, Vol. 4 (pp. 281-305). Iowa City: University of Iowa Press.

Kanwisher, N.G. (1987). Repetition blindness: Type recognition without token individuation. Cognition, 27, 117-143.

Lashley, K. (1951). The problem of serial order in behavior. In. L.A. Jeffress (Ed.), Cerebral mechanisms in behavior: The Hixon symposium (pp. 112-136). New York: Wiley.

Lerdahl, F., \& Jackendoff, R. (1983). A generative theory of tonal music. Cambridge, MA: MIT Press.

Levelt, W.J.M. (1983). Monitoring and self-repair in speech. Cognition, 14, 41-104.

Levelt, W.J.M. (1989). Speaking: From intention to articulation. Cambridge, MA: MTT Press.

MacKay, D.G. (1969). The repeated letter effect in the misspellings of normals and dysgraphics. Perception $\&$ Psychophysics, 5, 102-106.

MacKay, D.G. (1982). The problems of flexibility, fluency, and speed-accuracy trade-off in skilled behavior. Psychological Review, 89, 483-506.

MacKay, D.G. (1987). The organization of perception and action: A theory for language and other cognitive skills. New York: Springer.

MacKay, D.G., \& Miller, M.D. (1994). Semantic blindness: repeated concepts are difficult to encode and recall under time pressure. Psychological Science, 5, 52-55.

MacKay, D.G., \& Miller, M.D. (1994). Repetition deafness: Repeated words in computer-compressed speech are difficult to encode and recall. Psychological Science, S, 47-51.

Narmour, E. (1990). The analysis and cognition of basic melodic structures. Chicago: University of Chicago Press.

Norman, D.A. (1981). Categorization of action slips. Psychological Review, 88, 1-15.

Palmer, C. (1989). Mapping musical thought to musical performance. Joumal of Experimental Psychology: Human Perception and Performance, 15, 331-346.

Palmer, C. (1996). On the assignment of structure in music performance. Music Perception, 14, 21-54.

Palmer, C. (1997) Music performance. Annual Review of Psychology, 48, 115-138.

Palmer, C., \& Holleran, S. (1994). Harmony, melody, and frequency height influences in the perception of multivoiced music. Perception \& Psychophysics, 56, 301-312.

Palmer, C., \& van de Sande, C. (1993). Units of knowledge in music performance. Joumal of Experimental Psychology: Learning, Memory, and Cognition, 19, 457-470.

Palmer, C., \& van de Sande, C. (1995). Range of planning in music performance. Journal of Experimental Psychology: Human Perception and Performance, 21, 947-962.

Rasch, R.A. (1979). Synchronization in performed ensemble music. Acustica, 43, 121-131.

Repp, B. (1996). The art of inaccuracy? Why pianists' errors are 
difficult to hear. Music Perception, 14, 161-184.

Rumelhart, D.E., \& Norman, D.A. (1982). Simulating a skilled typist: A study of skilled cognitive-motor performance. Cognitive Science, 6, 1.36.

Seashore, C.E. (1938). Psychology of music. New York: McGraw-Hill.

Shaffer, L.H. (1976). Intention and performance. Psychological Review, 83, 375-393.

Shattuck-Hufnagel, S. (1979). Speech errors as evidence for a serial-ordering mechanism in sentence production. In W.E. Cooper \& C.T. Walker (Eds.), Sentence processing: Psycholinguistic studies presented to Merrill Garrett. Hillsdale, NJ: Erlbaum.

Sloboda, J.A., Davidson, J.W., Howe, M.J.; \& Moore, D.G. (1996). The role of practice in the development of performing musicians. British Journal of Psychology, 87, 287-309.

Sloboda, J.A., \& Howe, M.J.A. (1991). Biographical precursors of musical excellence: An interview study. Psychology of Music, 19, 3-21.

Sosniak, L.A. (1985). Learning to be a concert pianist. In B.S. Bloom (Ed.), Developing talent in young people (pp. 19-67). New York: Ballantine.

Stemberger, J.P. (1989). Speech errors in early child language production. Journal of Memory and Language, 28, 164-188.

Suzuki, S. (1983). Nurtured by love: The classic approach to talent education (second ed.). Smithtown, NY: Exposition Press.

\section{Appendix}

BEGINNERS' MUSICAL PIECES

Victorian Music Box (Wells)

Stuck on C (Vandall)

Alouette in C (Weybright ed)

Lavender's Blue (Schaum Ed)

Kumbayah (Broutman ed)

America (Carey \& Smith)

Minuet in G- 1 (J.S. Bach)

Minuet in G- 2 (J.S. Bach)

Christmas-Day Secrets (Dutton)

Musette in D-minor (Anon)

The Animal Band (Weybright ed)

Pop goes the weasel (Poole ed)

Mountain Dance (George)

Secondo (George)

Stomp Dance (Weybright ed)

Finale in C (Mozart)

Forest Green (Anonymous)

Beautiful Heaven (Fernandez)

Dangerous Journey (Koelling)

The Cuckoo (Daquin)

Chocolate Soldier (Cobb)

Roses for you (Curcio)
Sailing (Hurby ed)

Little Brown Jug (Stanley ed)

Black Forest Polka (Anon)

Light and Blue (Weybright ed)

Prelude (Glover)

Slow Dance (Graupner)

The Surf (J S Bastien)

Russian Folk Song (Beethoven)

INT'ERMEDIATES' MUSICAL PIECES

Clowns (Coffey)

The Pink Panther (Mancini)

Sonatina, Op. 36 No. 1, mvmt 2 (Clementi)

Sonatina, Op. 36 No. 1, mvmt 3 (Clementi)

Ecossaise (Beethoven) (2)

Musette (Bach)

Rondo (Mozart)

Home on the Range (Anonymous)

Sonatina, Op. 36 No. 3, mvmt 3 (Clementi)

Arietta (Mozart)

Sonatina, Op. 55 No. 1, mvmt 1 (Kuhlau)

Ecossaise (Hummel)

Country Dance (De Vito)

Sonatina, Op. 36 No. 2, mvmt 2 (Clementi)

Romance in G (Beethoven)

Theme in C (Beethoven-Arr.)

Sonatina Op. 36 No. 3 mvmt 1 (Clementi)(2)

The Happy Farmer (Schumann)

Arabesque (Bergmuller)

Sonatina in GM, mvmt 2 (Beethoven)

Sonatina in GM, mvmt 1 (Beethoven)

Petite Etude (Riegger)

MacGyver (Edelman)

Fur Elise (Beethoven) (2)

Arietta (Mozart)

ADULTS' MUSICAL PIECES

Waltz, Opus $70 \# 2$ (Chopin)

Nocturne, Opus 72 \#1 (Chopin)

Sonata in A-flat, mvmt 1 (Haydn)

Sonata Opus 35, mvmt 4 (Chopin)

Consolations I (Liszt)

Consolations II (Liszt)

Italian Concerto, mvmt 2 (Bach)

Italian Concerto, mvmt 3 (Bach)

Prelude, Opus 16 \#1 (Scriabin)

Prelude, Opus 16, \#2 (Scriabin)

Sonata, K596, mvmt 2 (Mozart)

Prelude, G Major (Chopin)

Prelude, le Tombeau de Couperin (Ravel)

Menuet, G-Major (Ravel)

Sonata, K282, mvmt 1 (Mozart)

Fantasie, Opus 17 (Liszt) 
Impromptu, Opus 142 \#3 (Schubert)

Prelude, Opus 23 \#4 (Rachmaninoff)
Prelude, le Tombeau de Couperin (Ravel)

Forlane, le Tombeau de Couperin (Ravel) 